\title{
Symmetry energy of warm nuclear systems
}

\author{
B. K. Agrawal ${ }^{1}$, J. N. De ${ }^{1}$, S. K. Samaddar ${ }^{1}$, M. Centelles ${ }^{2}$ and X. Viñas ${ }^{2}$ \\ ${ }^{1}$ Saha Institute of Nuclear Physics, \\ 1/AF Bidhannagar, Kolkata 700064, India \\ ${ }^{2}$ Departament d'Estructura $i$ Constituents de la Matèria, \\ Facultat de Física, \\ and Institut de Ciències del Cosmos, \\ Universitat de Barcelona, \\ Diagonal 645, 08028 Barcelona, Spain
}

\begin{abstract}
The temperature dependence of the symmetry energy and symmetry free energy coefficients of infinite nuclear matter and of finite nuclei is investigated. For infinite matter, both these coefficients are found to have a weaker dependence on temperature at densities close to saturation; at low but homogeneous densities, the temperature dependence becomes stronger. For finite systems, different definitions of symmetry energy coefficients are encountered in the literature yielding different values. A resolution to this problem is suggested from a global liquid-drop-inspired fit of the energies and free energies of a host of nuclei covering the entire periodic table. The hot nucleus is modeled in a subtracted finite-temperature-Thomas-Fermi framework, with dynamical surface phonon coupling to nucleonic motion plugged in. Contrary to infinite nuclear matter, a substantial change in the symmetry energy coefficients is observed for finite nuclei with temperature.
\end{abstract}

PACS numbers: 21.10.Dr,21.30.Fe,21.65.Ef,26.30.-k Keywords:

\section{INTRODUCTION}

The nuclear symmetry energy is a measure of the energy gain in converting isospin asymmetric nuclear matter to a symmetric system. Its value depends on density and temperature. It is assessed through the symmetry energy coefficient $e_{\text {sym }}(\rho, T)$; for an asymmetric system with density $\rho$, temperature $T$ and asymmetry $\delta$, the energy per nucleon, to a good approximation, can be written as

$$
e(\rho, \delta, T)=e(\rho, \delta=0, T)+e_{s y m}(\rho, T) \delta^{2},
$$

where $e_{\text {sym }}(\rho, T) \delta^{2}$ is the symmetry energy content per nucleon of the system and $e(\rho, \delta=0, T)$ is the energy per nucleon of symmetric nuclear matter. Here $\delta=\left(\rho_{n}-\right.$ $\left.\rho_{p}\right) /\left(\rho_{n}+\rho_{p}\right)$ is the isospin asymmetry, $\rho_{n}$ and $\rho_{p}$ are the neutron and proton densities of the system with $\rho=$ $\rho_{n}+\rho_{p}$. The coefficient $e_{\text {sym }}(\rho, T)$ can then be defined, without loss of generality, as

$$
e_{\text {sym }}(\rho, T)=\frac{1}{2}\left(\frac{\partial^{2} e(\rho, \delta, T)}{\partial \delta^{2}}\right)_{\delta=0} .
$$

The density dependence of the symmetry energy is instrumental in understanding the behaviour of the equation of state (EOS) of asymmetric nuclear matter. Accurate knowledge of this EOS is important in interpreting the structure of finite nuclei away from the stability line, some critical issues in astrophysics could only be addressed with this knowledge. Some progress has recently been made in understanding the behaviour of the symme- the isospin diffusion data in heavy ion collision [1 4] and from the available data for the neutron skin thickness of several nuclei [5]. The experimental data on the isotopic dependence of the nuclear giant monopole resonance in even-A Sn isotopes [6, 7] also provide some informations on the nuclear symmetry energy which are in agreement with those derived from the analyses of the isospin diffusion data. The behaviour of the nuclear symmetry energy at supranormal densities is largely unknown. The precise measurements of the observable properties of compact stars and the transport model analyses of heavy-ion collisions at intermediate and high energies may provide some constraints on the high density behaviour of the symmetry energy.

Understanding the thermal behavior of the symmetry energy is of utmost contemporary importance. It has a role in changing the nuclear drip lines as the nuclei warm up. It is a key element in deciding a number of serious issues in the astrophysical scenario like supernova explosions 8] or explosive nucleosynthesis. A large (small) value of the symmetry coefficient, say, inhibits (accelerates) the change of protons to neutrons through electron capture [9, 10]. The consequent modulation of the EOS of hot nuclear matter through shift in isospin asymmetry shapes the dynamical phases of the collapse and explosion of a massive star. Furthermore, in this rapidly changing scenario, the hot and possibly dilute nuclear matter is an inhomogeneous congregate of nuclear clusters of different sizes. A precision understanding of the thermal evolution of the symmetry coefficients of finite nuclei then becomes 
tive $L$ of the symmetry coefficient $e_{\text {sym }}$

$$
L=\left.3 \rho_{0} \frac{\partial e_{\text {sym }}}{\partial \rho}\right|_{\rho_{0}}
$$

reflects the pressure difference on the neutrons and protons and is thus one of the determinants in fixing the neutron skin of nuclei [11], the nature and stability of phases within a warm neutron star, its crustal composition or its thickness [12] would be strongly influenced from the temperature dependence of symmetry energy. The cooling rate of warmer proto-neutron stars through neutrino convection [13] may also be affected from the thermal change of the symmetry energy.

A few measurable bulk parameters often help in giving a better feel on the characteristics of the nuclear EOS at zero temperature around the saturation density. At $T=0$, the first term in Eq. (1) can be cast as

$$
e(\rho, \delta=0) \simeq a_{v}+\frac{1}{2} K_{v} \epsilon^{2},
$$

and the symmetry coefficient is written as

$$
e_{\text {sym }}(\rho) \simeq e_{\text {sym }}\left(\rho_{0}\right)-L \epsilon+\frac{1}{2} K_{\text {sym }} \epsilon^{2},
$$

where $\epsilon=\left(\rho_{0}-\rho\right) / 3 \rho_{0}, a_{v}$ is the energy per nucleon of symmetric nuclear matter, $K_{v}$ is the isoscalar density incompressibility and $K_{\text {sym }}$ is the symmetry incompressibility, all at saturation density $\rho_{0}$. The parameters $a_{v}, \rho_{0}$, or $K_{v}$ are known more or less in tight bounds [14], while $L$ and $K_{\text {sym }}$ are less precise [4, [5, 15]. Mindful that the above characterization of the EOS is valid only close to the saturation density, the EOS of infinite homogeneous nuclear matter spanning a wide density range has been calculated with an effective interaction as input, the interaction designed so as to describe broadly the experimental observables quite well. The symmetry energies at different temperatures and densities have then been calculated $16-19]$.

The above calculations for homogeneous nuclear matter have been done in the mean-field (MF) approximation. Low density nuclear matter is, however, not homogeneous. Formation of clusters of different sizes [20, 21] becomes energetically more favorable there. A detailed knowledge of the composition of nuclear matter is then needed to appreciate how the energies and symmetry energies are affected because of clusterization. In hot inhomogeneous supernova matter, the neutrino-driven energy transfer [10] is dictated partly by the size of the clusters. Using the virial expansion technique, clusterization in dilute nuclear matter and its import in the evaluation of the symmetry energy and its temperature dependence have been investigated by Horowitz and coworkers [22, 23], where they consider matter to be composed of only very light particles whose scattering phase-shifts are known. The calculations are later extended with inclusion of all of the grand-canonical partition function for nuclear matter in the $S$-matrix framework [24 26]. One interesting fallout of this calculation is that even symmetric nuclear matter, when dilute and warm, may have sizeable symmetry energy content; this is so because the produced clusters may not be all symmetric, though the disassembled matter conserves overall vanishing isospin asymmetry. The persistence of this feature in clusterized dilute matter makes the definition of the symmetry energy coefficient $e_{\text {sym }}$ or the symmetry free energy coefficient $f_{\text {sym }}$ ambiguous. The symmetry coefficient $e_{\text {sym }}$ as defined by Eq.(2) has been approximated in the literature in several ways:

$$
\begin{aligned}
& e_{\text {sym }}(\rho, T)=[e(\rho, \delta, T)-e(\rho, \delta=0, T)] / \delta^{2}, \\
& e_{\text {sym }}(\rho, T)=e(\rho, \delta=1, T)-e(\rho, \delta=0, T),
\end{aligned}
$$

For homogeneous nuclear matter at densities upto saturation, $e(\rho, \delta, T)$ is found to be linear in $\delta^{2}$ in the whole range of $\delta$. All the three definitions (given by Eqs. (2), (6) and (7)) yield nearly the same value of $e_{\text {sym }}$. The total energy or free energy of the asymmetric dilute inhomogeneous system, however, deviates strongly from the abovementioned linearity. This leads to different values [27] for $e_{\text {sym }}$ or $f_{\text {sym }}$ from the three definitions. Definition given by Eq. (7) has been used [28, 29] in the experimental determination of the values of the symmetry coefficients of warm dilute nuclear matter.

For application in core-collapse supernova simulations, it is more insightful to have understanding about the temperature dependence of the symmetry energy of isolated nuclei or nuclei embedded in a nucleonic medium [30]. In a limited temperature domain $(T \leq 2 \mathrm{MeV})$, calculations of the symmetry energy coefficients of atomic nuclei have been attempted in a schematic model by Donati et al., 31]. These calculations take into account the coupling of the nucleons to the dynamical surface phonons. This results in an increased effective nucleon mass, the so-called energy mass (or $\omega$-mass $m_{\omega}$ ) coming into play. The $\omega$ mass carries signatures of interaction that are nonlocal in time 32. The energy mass decreases with temperature [33], this brings in a decreased density of states and thus an increase in the symmetry coefficient. A fall-out is that electron captures are hindered in supernova matter. From shell-model Monte-Carlo calculations in this limited temperature range, quantitative support to these findings were given by Dean et al., 34]. The symmetry coefficients so calculated are, however, much below the nominally acceptable range. Calculations have also been done recently by Lee and Mekjian [35] in a density functional theoretic approach, but the suitability of the approximations used keeps the calculations trustworthy only in a low temperature domain $(T \leq 3 \mathrm{MeV})$.

Very recently, attempts have been made to explore the thermal evolution [36] of the symmetry energy coefficients of specific atomic masses in an extended temperature region $(T \leq 8 \mathrm{MeV})$ in the finite- 
subtraction technique [37]. For a finite nucleus of mass $A$, the symmetry coefficient is defined as in Eq. (6) using the difference of the nuclear parts of the energy per nucleon of a nuclear pair of mass number $A$ having different isospins. The definition from this difference method [34], however, does not prescribe a unique value of the symmetry coefficient for the nucleus; the values depend on the choice of the isospin asymmetric isobar pair. Another definition [18] based on the local density approximation (LDA) has also been used. It has its drawback too, it suffers from its dependence on the isospin of the nucleus and also on the charge-dependent density distribution $\rho(r)$.

An unambiguous definition of the temperature dependent symmetry energies and the symmetry coefficients can, however, be obtained in the framework of the liquid drop model. Very recently, the energies and free energies of a large number of nuclei spanning almost the entire periodic table were calculated [38] as a function of temperature in the subtracted FTTF method and fitted in the spirit of the liquid drop model with volume, surface, Coulomb and symmetry energy coefficients. The temperature-dependent coefficients (including the symmetry coefficients) so obtained convey a sense of average, but they are ambiguity-free and in the present state of our knowledge serves the purpose.

The article is organized as follows. In Sec. II, we portray the theoretical outlines. Sec. IIA discusses the EOS of homogeneous nuclear matter. Hot nuclear systems are inherently unstable, a suitable model for proffering stability to such systems is described in Sec. IIB; deriving relevant observables from such a description is also briefly outlined there. A short discussion on the energy mass is given in Sec. IIC, relevant formulas for calculating the symmetry coefficients are presented in Sec IID. In Sec. III, results and discussions are given. Sec. IV ends with the concluding remarks.

\section{ELEMENTS OF THEORY}

The methodology employed to calculate the symmetry energy and symmetry free energy of hot infinite and finite nuclear systems is described in this section. Selfconsistent thermal models are employed. Three interactions of the Skyrme class, namely, SkM* 39], SLy4 [40], and SK255 41] are used to describe the systems. Ground state bulk properties of nuclei over the whole periodic table for $A \geq 16$ are reproduced quite satisfactorily with these interactions. Some bulk properties like the saturation density, symmetry energy coefficient, isoscalar incompressibility, etc, for these interactions are given in Table I. The interaction SLy4 describes systems with extreme isospin better, SK255 gives comparatively larger symmetry energy coefficient and larger incompressibility. Different interactions are chosen to see whether different thermal sensitivity of the symmetry properties becomes manifest even if the ground-state energies are described

\section{A. Energy density functionals}

With the Skyrme energy density functional, the total energy density of the system is written as,

$$
\begin{aligned}
\mathcal{E}(r)= & \frac{\hbar^{2}}{2 m_{n, k}} \tau_{n}+\frac{\hbar^{2}}{2 m_{p, k}} \tau_{p} \\
& +\frac{1}{2} t_{0}\left[\left(1+\frac{1}{2} x_{0}\right) \rho^{2}-\left(x_{0}+\frac{1}{2}\right)\left(\rho_{n}^{2}+\rho_{p}^{2}\right)\right] \\
& +\frac{1}{12} t_{3} \rho^{\alpha}\left[\left(1+\frac{x_{3}}{2}\right) \rho^{2}-\left(x_{3}+\frac{1}{2}\right)\left(\rho_{n}^{2}+\rho_{p}^{2}\right)\right] \\
& +\frac{1}{16}\left[3 t_{1}\left(1+\frac{1}{2} x_{1}\right)-t_{2}\left(1+\frac{1}{2} x_{2}\right)\right](\nabla \rho)^{2} \\
& -\frac{1}{16}\left[3 t_{1}\left(x_{1}+\frac{1}{2}\right)+t_{2}\left(x_{2}+\frac{1}{2}\right)\right]\left[\left(\nabla \rho_{n}\right)^{2}+\left(\nabla \rho_{p}\right)^{2}\right] \\
& +\mathcal{E}_{c}(r) .
\end{aligned}
$$

In Eq. (8), $t_{0}, t_{1}, t_{2}, t_{3}, x_{0}, x_{1}, x_{2}, x_{3}$ and $\alpha$ are the Skyrme parameters (given in Table II for the interactions) and $\rho_{n}$ and $\rho_{p}$ are the neutron and proton densities $\left(\rho=\rho_{n}+\rho_{p}\right)$. For infinite homogeneous systems, the derivative terms do not contribute, neither the Coulomb term $\mathcal{E}_{c}$, since the whole system is charge neutral. The finite nucleus has contributions from the Coulomb force though, as it is charged. The nucleon effective mass $m_{q, k}$ is defined through

$$
\begin{aligned}
\frac{m}{m_{q, k}(r)}= & 1+\frac{m}{2 \hbar^{2}}\left\{\left[t_{1}\left(1+\frac{x_{1}}{2}\right)+t_{2}\left(1+\frac{x_{2}}{2}\right)\right] \rho\right. \\
& \left.+\left[t_{2}\left(x_{2}+\frac{1}{2}\right)-t_{1}\left(x_{1}+\frac{1}{2}\right)\right] \rho_{q}\right\}
\end{aligned}
$$

with $q=(n, p)$ referring to neutrons or protons. This effective mass, often called the nucleon $k$-mass, arises from the momentum dependence in the effective interaction. In the Thomas-Fermi approximation at finite tempertature, the kinetic energy density is

$$
\tau_{q}=\frac{2 m_{q, k}}{\hbar^{2}} A_{q}^{*} T J_{3 / 2}\left(\eta_{q}\right),
$$

with

$$
A_{q}^{*}=\frac{1}{2 \pi^{2}}\left(\frac{2 m_{q, k} T}{\hbar^{2}}\right)^{3 / 2} .
$$

The function $J_{k}(\eta)$ is the standard Fermi integral. At zero temperature, $\tau_{q}$ reduces to the familiar expression,

$$
\tau_{q}=\frac{3}{5}\left(3 \pi^{2}\right)^{2 / 3} \rho_{q}^{5 / 3}
$$

The fugacity $\eta_{q}$ is obtained as,

$$
\eta_{q}(r)=\left[\mu_{q}-V_{q}(r)\right] / T
$$

where $V_{q}(r)$ is the single-particle potential experienced by the nucleons (including the Coulomb part for the protons in finite systems) and $\mu_{q}$ their chemical potentials. The nucleonic density $\rho_{q}$ is related to $\eta_{q}$ by 
The Coulomb energy density is given by $\mathcal{E}_{c}(r)=\mathcal{E}_{c}^{d}(r)+$ $\mathcal{E}_{c}^{e x}(r)$, where the direct contribution is

$$
\mathcal{E}_{c}^{d}(r)=\frac{1}{2} \rho_{p}(r) \int \rho_{p}\left(r^{\prime}\right) \frac{e^{2}}{\left|\mathbf{r}-\mathbf{r}^{\prime}\right|} d \mathbf{r}^{\prime}
$$

and the exchange part, in the Slater approximation, is

$$
\mathcal{E}_{c}^{e x}=-\frac{3 e^{2}}{4 \pi}\left(3 \pi^{2}\right)^{1 / 3} \rho_{p}^{4 / 3}(r) .
$$

In the Landau quasi-particle approximation, the entropy density of the nucleons can be computed as

$$
\mathcal{S}(r)=-\frac{2}{h^{3}} \sum_{q} \int\left[n_{q} \ln n_{q}+\left(1-n_{q}\right) \ln \left(1-n_{q}\right)\right] d \mathbf{p}(17)
$$

which simplifies to

$$
\mathcal{S}(r)=\sum_{q}\left[\frac{5}{3} J_{3 / 2}\left(\eta_{q}\right) / J_{1 / 2}\left(\eta_{q}\right)-\eta_{q}\right] \rho_{q} .
$$

The $n_{q}$ 's in Eq. (17)are the single-nucleon distributions; they are given by

$$
n_{q}(r, p, T)=\left[1+\exp \left\{\frac{p^{2}}{2 m_{q, k} T}-\eta_{q}\right\}\right]^{-1} .
$$

The nucleon density $\rho_{q}(r)$ (given by Eq. (14)) is obtained from the momentum integration of the distribution function. Once the entropy density is known, the free energy density $\mathcal{F}(r)$ of the system is obtained as

$$
\mathcal{F}(r)=\mathcal{E}(r)-T \mathcal{S}(r)
$$

For an infinite system, the energy and free energy per nucleon are then calculated as $e=\mathcal{E} / \rho$ and $f=\mathcal{F} / \rho$. The symmetry coefficient $e_{\text {sym }}(\rho, T)$ can then be computed from Eq. (2). The coefficient $f_{\text {sym }}(\rho, T)$ can be similarly calculated.

\section{B. Modeling the hot nucleus}

The nucleon density profile for the hot nucleus is computed self-consistently in the finite temperature ThomasFermi (FTTF) approach. Calculations in a box, as is usually done in the Thomas-Fermi procedure, however, lead to difficulties. At large distances from the center, the density is small, the single-particle potential $V_{q} \sim 0$, $\eta_{q}<<0$, and then $\rho_{q} \sim e^{\mu_{q} / T}$, a constant. At large distances from the nuclear center, the particle density then does not vanish (at $T=0$, there are no problems though, $\mu_{q}$ being negative, $\rho_{q}=0$ at large distance). The pressure of the system is then nonzero, making the system thermodynamically unstable. The density profile also depends on the size of the box in which the FTTF calculations are performed. The problem is overcome in the subtraction procedure [37, 42], where the hot nucleus, assumed to be gas representing the evaporated nucleons from the hot nucleus, is extracted from the embedding environment. This method is based on the existence of two solutions to the FTTF equations, one corresponding to the liquid phase with the surrounding gas $(l g)$ and the other corresponding to the gas phase $(g)$ alone [43]. The density of the thermalized nucleus in equilibrium is given by $\rho_{q}=\rho_{l g}^{q}-\rho_{g}^{q}$. It is independent of the box size, also goes to zero at large distances implying a vanishing surface pressure. The nucleon number conservation gives

$$
\int\left[\rho_{l g}^{q}(r)-\rho_{g}^{q}(r)\right] d^{3} r=N_{q}
$$

where $N_{q}$ is the number of neutrons or protons in the nucleus. The total energy $E$ of the nucleus is given by

$$
E=E_{l g}-E_{g}
$$

where $E_{l g}$ and $E_{g}$ are the total energies of the liquid-gas system and of the gas alone. From Eq. (17), the total entropy of the nucleus can be recast as [44],

$S=-\sum_{q} \int g_{q}\left(\epsilon_{q}, T\right)\left[f_{q} \ln f_{q}+\left(1-f_{q}\right) \ln \left(1-f_{q}\right)\right] d \epsilon_{q}(23)$

where $f_{q}$ is the single-particle occupation function in the energy space,

$$
f_{q}\left(\epsilon_{q}, \mu_{q}, T\right)=\left[1+\exp \left(\epsilon_{q}-\mu_{q}\right) / T\right]^{-1}
$$

and $g_{q}$ is the subtracted single-particle level density. It is given as [45]

$$
\begin{aligned}
g_{q}\left(\epsilon_{q}, T\right)= & \frac{4 \sqrt{2}}{\pi \hbar^{3}} \int\left[\left(m_{q, k}^{l g}\right)^{3 / 2} \sqrt{\epsilon_{q}-V_{q}^{l g}(r)}\right. \\
& \left.-\left(m_{q, k}^{g}\right)^{3 / 2} \sqrt{\epsilon_{q}-V_{q}^{g}(r)}\right] r^{2} d r .
\end{aligned}
$$

The free energy is calculated from $F=E-T S$. In terms of the occupation function, the density in the FTTF approximation can be written as

$$
\begin{aligned}
\rho_{q}^{i}(r)= & \frac{1}{2 \pi^{2} \hbar^{3}}\left(2 m_{q, k}^{i}(r)\right)^{3 / 2} \\
& \times \int \sqrt{\epsilon_{q}-V_{q}^{i}(r)} f_{q}\left(\epsilon_{q}, \mu_{q}, T\right) d \epsilon_{q} .
\end{aligned}
$$

Here $i$ stands for the liquid-gas phase or the gas phase.

\section{The energy mass}

The coupling of the nucleons to the dynamical surface phonons results in an increased effective mass, with appearance of the so-called energy mass $m_{\omega}$, as stated earlier. This effective mass decreases with temperature, 
Taking the energy mass into consideration, the total effective mass of the nucleon $m^{*}$ can be written as

$$
m^{*}=m\left(\frac{m_{k}}{m}\right)\left(\frac{m_{\omega}}{m}\right),
$$

where $m$ is the nucleon mass (for simplicity, we take the neutron-proton mass difference to be zero as this is very small compared to their masses). Aside from being temperature-dependent, $m_{\omega}$ is density-dependent; for a finite nucleus, its value therefore varies with position. A fully self-consistent calculation of $m_{\omega}$ is thus very involved and not within the scope of the present work. We therefore take a phenomenological form [46] for $m_{\omega}$ such that

$$
\frac{m_{\omega}}{m}=1.0-0.4 A^{1 / 3} \exp \left[-\left(\frac{T}{21 A^{-1 / 3}}\right)^{2}\right] \frac{1}{\rho(0)} \frac{d \rho(r)}{d r} .
$$

The temperature $T$ and the distance $r$ are measured in $\mathrm{MeV}$ and fm units, respectively, $\rho(0)$ is the central density of the nucleon distribution in the nucleus. The density in the above equation is $\rho(r)=\rho_{l g}(r)-\rho_{g}(r) ; A$ refers to the mass number of the subtracted density (hereafter called the liquid mass number). The collectivity implied in $m_{\omega}$ refers to the liquid phase only, meaning thereby $m_{\omega}^{l g}=m_{\omega}$ and $m_{\omega}^{g}=m$.

The self-consistent calculation of the density profile with the inclusion of $\omega$-mass is complex; a realistic extension of the method given in Ref. [46 is therefore adopted. This is described in detail in Ref. [44].

The subtracted level density corresponding to Eq. (25) is now modified as

$$
\begin{aligned}
\tilde{g}_{q}\left(\epsilon_{q}, T\right)= & \frac{4 \sqrt{2}}{\pi \hbar^{3}} \int\left[\left(m_{q, k}^{l g} \frac{m_{\omega}}{m}\right)^{3 / 2} \sqrt{\epsilon_{q}-V_{q}^{l g}(r) \frac{m}{m_{\omega}}}\right. \\
& \left.-\left(m_{q, k}^{g}\right)^{3 / 2} \sqrt{\epsilon_{q}-V_{q}^{g}(r)}\right] r^{2} d r .
\end{aligned}
$$

The densities in the $l g$ or $g$ phase modify accordingly

$$
\begin{aligned}
\tilde{\rho}_{q}^{i}(r)= & \frac{1}{2 \pi^{2} \hbar^{3}}\left[2 m_{q, k}^{i} \frac{m_{\omega}}{m}\right]^{3 / 2} \int \sqrt{\epsilon_{q}-V_{q}^{i} \frac{m}{m_{\omega}^{i}}} \\
& \times f_{q}\left(\epsilon_{q}, \tilde{\mu}_{q}, T\right) d \epsilon_{q} .
\end{aligned}
$$

The chemical potential $\mu_{q}$ modifies to $\tilde{\mu}_{q}$ to conserve the particle number in the nucleus

$$
N_{q}=\int \tilde{g}_{q}\left(\epsilon_{q}, T\right) f_{q}\left(\epsilon_{q}, \tilde{\mu}_{q}, T\right) d \epsilon_{q} .
$$

The energy and the free energy of the nucleus is calculated with the modified density given by Eq. (30).

\section{The symmetry coefficients}

For homogeneous infinite matter, we have calculated the symmetry energy coefficients using Eq. (7). The symmetry free energy coefficients are likewise calculated,
The kinetic $(K)$ and potential $(I)$ components of the symmetry coefficients $e_{\text {sym }}^{K}, e_{\text {sym }}^{I}, f_{\text {sym }}^{K}$ and $f_{\text {sym }}^{I}$ are similarly calculated replacing the energy $e$ and free energy $f$ (in Eq. (7) and (32)) by $e_{K}$ or $e_{I}$ and $f_{K}$ or $f_{I}$, respectively where $e_{K}, e_{I}$, say, stand for the kinetic and interaction parts of the energy per nucleon.

For a finite nucleus (with Coulomb interaction turned on), the symmetry energy coefficient in the difference method is defined as

$$
e_{\text {sym }}(A, T)=\left[e_{n}\left(A, I_{1}, T\right)-e_{n}\left(A, I_{2}, T\right)\right] /\left(I_{1}^{2}-I_{2}^{2}\right)(33)
$$

where the $e_{n}$ 's are the nuclear part of the energy per nucleon of the nuclear pair of mass $A$ having different isospins $I_{1}$ and $I_{2}$. In the local density approximation (LDA), one defines the symmetry energy coefficient for a specific nucleus once its density profile is known. It is given as

$$
e_{\text {sym }}(A, T)=\frac{1}{I^{2} A} \int \rho(r) e_{\text {sym }}[\rho(r), T] \delta_{l}^{2}(r) d^{3} r
$$

In Eq. (34), $e_{\text {sym }}[\rho(r), T]$ is the symmetry coefficient at temperature $T$ of infinite matter at the value of the local density $\rho(r), \rho_{n}(r)$ and $\rho_{p}(r)$ are the neutron and proton densities and $\delta_{l}(r)$ is the isospin asymmetry of the local density. The symmetry free energy coefficient can be defined exactly in a parallel way in the difference method and also in LDA.

As already discussed, both the difference method and the LDA fail to give unique values for the symmetry energies for finite nuclei or their temperature dependence. A possible means to arrive at unambiguous values of the temperature-dependent symmetry coefficients can be achieved in the framework of the liquid-drop model, exploiting the Bethe-Weizsäcker mass formula. One can calculate the energies and free energies of a set of nuclei spanning nearly the whole periodic table as a function of temperature in the subtracted FTTF procedure with effects from energy-mass taken into account and expand the energy or free energy in terms of macroscopic parameters as

$$
\begin{aligned}
E(N, Z, T)= & a_{v}(T) A+a_{s}(T) A^{2 / 3}+a_{c} \frac{Z^{2}}{A^{1 / 3}} \\
& +e_{\text {sym }}(A, T) I^{2} A, \\
F(N, Z, T)= & f_{v}(T) A+f_{s}(T) A^{2 / 3}+a_{c} \frac{Z^{2}}{A^{1 / 3}} \\
& +f_{\text {sym }}(A, T) I^{2} A .
\end{aligned}
$$

The coefficients $a_{v}, a_{s}$ and $a_{c}$ are the volume, surface and Coulomb energy coefficients; similarly $f_{v}$ and $f_{s}$ refer to the volume and surface free energies. The Coulomb energy and free energy are the same. Nuclei are finite systems, they have varying density profiles. This necessitates introduction of a mass-dependent surface component in $e_{\text {sym }}(A, T)$ over and above the massindependent volume component $e_{\text {sym }}^{v}(A, T)$. Two defini- 
in $e_{\text {sym }}(A, T)$. The first, hereafter referred to as I 47 49] is

$$
e_{\text {sym }}(A, T)=\frac{e_{\text {sym }}^{v}(T)}{1+\frac{e_{s y m}^{v}(T)}{\beta_{E}(T)} A^{-1 / 3}},
$$

and the second, referred to as II [50 52] is,

$$
e_{\text {sym }}(A, T)=e_{\text {sym }}^{v}(T)-e_{\text {sym }}^{s}(T) A^{-1 / 3} .
$$

In Eq. (38), the first term on the right hand side (rhs) measures the contribution from the nearly constant value of the density from the central part of the nucleus; at $T=0, e_{\text {sym }}^{v}$ is to be equated to $e_{\text {sym }}$ corresponding to infinite nuclear matter at saturation density. The second term in Eq. (38) is the surface symmetry energy, accounting for the contribution coming from the surface profile specific to the nuclear mass number. In Eq. (37), $\beta_{E}(T)$ is a measure of the surface symmetry energy. In the limit of large $A,\left(e_{\text {sym }}^{v}(T)\right)^{2} / \beta_{E}(T) \sim e_{\text {sym }}^{s}(T)$. The phenomenological value of $e_{\text {sym }}^{s}(T=0)$ is $\sim 50-58 \mathrm{MeV}$ [51 53] and that of $e_{\text {sym }}^{v}(T=0) / \beta_{E}(T=0)$ is in the close range of $\sim 2.4 \pm 0.4[48,49$, , 54].

Since the Coulomb energies are precisely known in a FTTF calculation, one can make a four-parameter fit of only the nuclear part of the energies $E_{n}$ and free energies $F_{n}$ of nuclei,

$E_{n}(N, Z, T)=a_{v}(T) A+a_{s}(T) A^{2 / 3}+e_{s y m}(A, T) I^{2} A(39)$

$F_{n}(N, Z, T)=f_{v}(T) A+f_{s}(T) A^{2 / 3}+f_{\text {sym }}(A, T) I^{2} A(40)$

Here, $e_{\text {sym }}(A, T)$ is given by Eq. (37) or (38). Similarly, $f_{\text {sym }}(A, T)$ is defined as,

$$
\begin{gathered}
f_{\text {sym }}(A, T)=\frac{f_{\text {sym }}^{v}(T)}{1+\frac{f_{\text {sym }}^{v}(T)}{\beta_{F}(T)} A^{-1 / 3}}, \\
f_{\text {sym }}(A, T)=f_{\text {sym }}^{v}(T)-f_{\text {sym }}^{s}(T) A^{-1 / 3} .
\end{gathered}
$$

The four-parameter set $f_{v}, f_{s}, f_{s y m}^{v}$ and $f_{s y m}^{s}\left(\right.$ or $\left.\beta_{F}\right)$ has the same connotation as the set $a_{v}, a_{s}, e_{\text {sym }}^{v}$ and $e_{\text {sym }}^{s}$ (or $\left.\beta_{E}\right)$, except that the former set refers to free energy.

\section{RESULTS AND DISCUSSIONS}

To study the temperature dependence of the symmetry coefficients of infinite and finite nuclear systems, we have used three interactions, all from the Skyrme class, namely $\mathrm{SkM}^{*}$, SLy4 and SK255. These interactions describe the ground state energies of finite nuclei rather well, there is some difference though in the computed values of some of the macroscopic observables as shown in Table I.

The symmetry coefficients $e_{\text {sym }}$ and $f_{\text {sym }}$ as calculated from Eq. (6) for infinite matter are displayed in Fig. 1

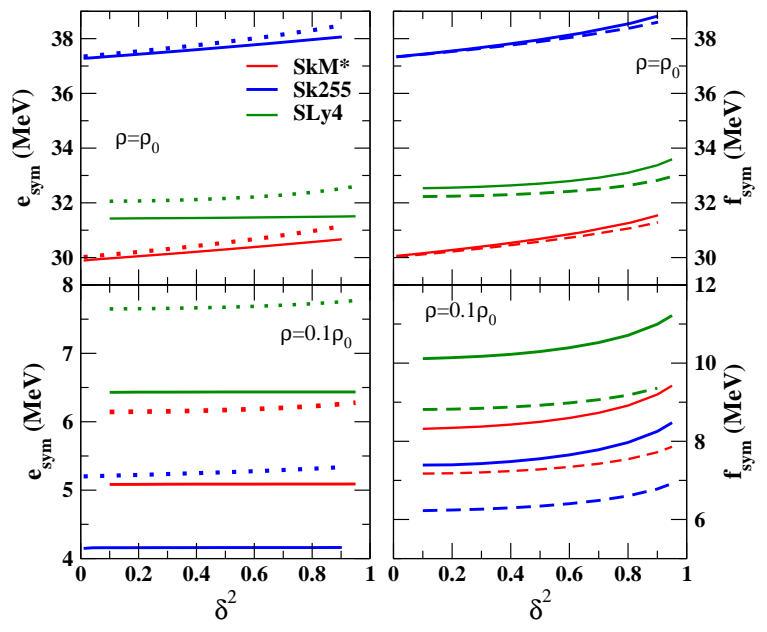

FIG. 1: (Color online) The symmetry energy coefficient $e_{\text {sym }}$ and symmetry free energy coefficient $f_{\text {sym }}$ of infinite nuclear matter as defined from Eq. (6) shown as a function of the asymmetry parameter $\delta^{2}$ at two densities $\rho=\rho_{0}$ and at $\rho=$ $0.1 \rho_{0}$ for three interactions, $\mathrm{SkM}^{*}$ (red), SK255 (blue) and SLy4 (green). The dotted lines refer to calculations at $T=$ $0.0 \mathrm{MeV}$, the dashed lines at $T=5.0 \mathrm{MeV}$ and the full lines at $T=8.0 \mathrm{MeV}$, respectively.

interactions at the corresponding saturation densities $\rho_{0}$ and at one-tenth of this density. The left panels refer to $e_{\text {sym }}$, the right panels to $f_{\text {sym }}$. The dotted lines represent calculations at $T=0$, the dashed lines at $T=5$ and full lines at $T=8 \mathrm{MeV}$, respectively. A non-zero slope at all densities and temperatures for most of the lines shows that the symmetry energy or symmetry free energy content in the system is nonlinear in $\delta^{2}$, but the small value of the slope (as seen from the slow rise of $e_{\text {sym }}$ or $f_{\text {sym }}$ with $\delta^{2}$ ) shows that the nonlinearity is weak. From now on, we use the definition as given by Eq. (7) for the symmetry coefficients for infinite nuclear matter.

The temperature dependence of the symmetry energy coefficients corresponding to the three interactions is displayed in Fig. 2. The upper panels pertain to the saturation density $\rho_{0}$, the lower panels to $\rho=\rho_{0} / 10$. At the saturation density, it is seen that $e_{\text {sym }}$, shown by the full lines (whose values are somewhat different for different interactions), has a very weak temperature dependence. The behavior for the interaction component $e_{\text {sym }}^{I}$ (the dashed lines) and the kinetic energy component $e_{\text {sym }}^{K}$ (shown by the dotted lines) are similar. It may be mentioned that the kinetic energy component is evaluated with the bare nucleon mass. At the subsaturation density, $e_{\text {sym }}$ shows a slow fall [16, 19, 36]. This is essentially due to the decrease in its kinetic energy part with temperature. The lesser importance of the Pauli blocking because of the increased diffuseness of the nucleon Fermi surfaces with rising temperature lies behind this decrement in $e_{s y m}^{K}$ at lower densities. At these densities, the symmetry free energy coefficient displays, 


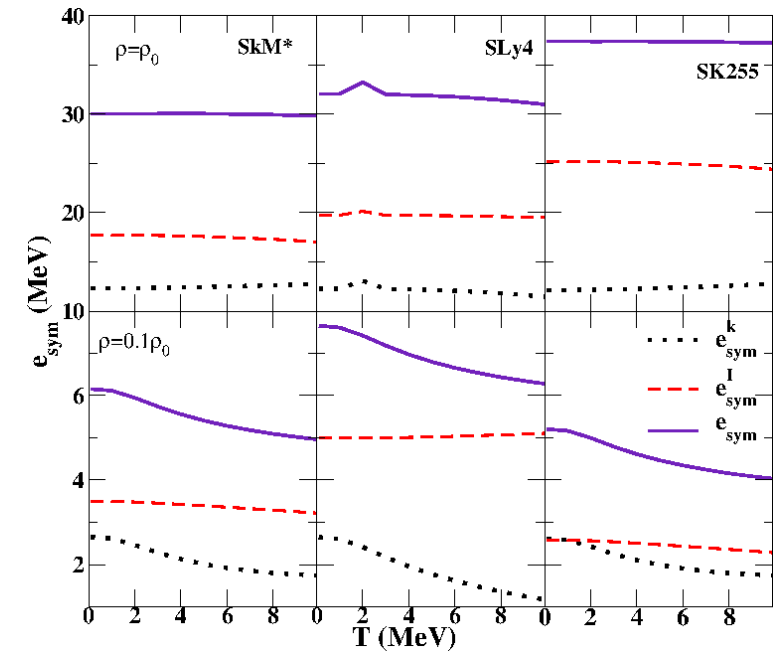

FIG. 2: (Color online) The thermal evolution of the symmetry energy coefficients $e_{\text {sym }}$ as defined in Eq.(7) and its interaction component $e_{s y m}^{I}$ and kinetic energy component $e_{s y m}^{K}$ shown at densities $\rho=\rho_{0}$ and at $\rho=0.1 \rho_{0}$ for the three interactions as shown. The full violet line corresponds to $e_{s y m}$, the black dotted line refers to $e_{s y m}^{K}$ and the red dashed line to $e_{\text {sym }}^{I}$.

seen from the lower panel of Fig. 3. This is in fair agreement with those obtained earlier [16, 36]. The increase in $f_{\text {sym }}$ with temperature at very low densities can be understood from the fact that $s_{\text {sym }}$, the symmetry entropy coefficient is then negative, $\sim-\frac{1}{2}$ [55]. Thus, even if $e_{\text {sym }}$ falls slower, the rise in $f_{\text {sym }}$ is noticeable at these densities. At higher density, $f_{\text {sym }}$ as with $e_{\text {sym }}$ shows nearly no trace of temperature dependence; this is shown in the upper panel of Fig. 3 for $\rho=\rho_{0}$.

In the previous section, we have already adumbrated that the symmetry energy coefficients of finite nuclei may not come out unique in the LDA (Eq. (34)) or in the difference method (Eq. (33)). As a demonstration, we show this in Figs. 4-6 where the thermal evolution of the symmetry coefficients has been calculated in LDA and in the difference method with the SLy4 interaction. In the panels of Fig. 4, the temperature dependence of $e_{\text {sym }}$, calculated in LDA for $A=56,150$ and 208 is displayed. The values of $e_{\text {sym }}$ decrease with temperature, but it is evident that for a given isobar, the symmetry energy coefficient depends on the choice of the $(Z, N)$ value, the isobar with the higher atomic number having larger symmetry energy. Figs. 5 and 6 show $e_{\text {sym }}$ and $f_{\text {sym }}$ evaluated in the difference method for the nuclei $A=56$ and $A=112$ taken as representative examples. The results again are seen to depend on the choice of the nuclear pairs (shown by the proton numbers in brackets) for the isobars. The decrease in $e_{\text {sym }}$ (Fig. 4 and upper panels of Figs. 5 and 6 ) or the increase in $f_{\text {sym }}$ (lower panels of Figs. 5 and $6)$ with temperature for finite nuclei comes from the increased weight from the temperature-dependent surface

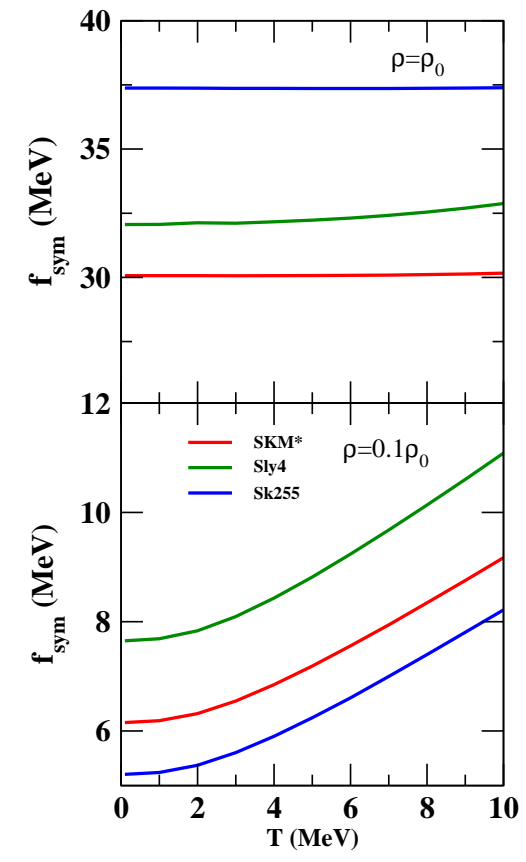

FIG. 3: (Color online) The temperature dependence of the symmetry free energy coefficient of infinite nuclear matter at two densities $\rho=\rho_{0}$ and at $\rho=0.1 \rho_{0}$ as shown for the three interactions, $\mathrm{SkM}^{*}$ (red line), SLy4 (green line) and SK255 (blue line).

As already stated, unique values of symmetry coefficients could be ascribed to finite nuclei and their temperature dependence obtained, albeit in an average sense, making use of the Bethe-Weizsäcker mass formula. To this end, a set of spherical and near-spherical nuclei covering almost the entire periodic table is chosen. We take 69 nuclei, having masses as $36 \leq A \leq 218$ and atomic charges $14 \leq Z \leq 92$ (the list of nuclei is taken from Ref. 56]). Their energies and free energies are then calculated in a temperature grid in the subtracted FTTF procedure, taking into account the dressing of the nucleon mass to energy mass $m_{\omega}$ arising from the coupling of the nucleonic motion to the surface vibrations. The nuclear part of the energies and free energies (i.e., the Coulomb energy is subtracted from the total energies or free energies) are then fitted with a four-parameter fit. The parameter sets are $\left(a_{v}, a_{s}, e_{s y m}^{v}\right.$ and $\left.\beta_{E}\right)$ or $\left(f_{v}, f_{s}, f_{s y m}^{v}\right.$ and $\beta_{F}$ ) for energies or free energies in definition I. In definition II, the parameters are $\left(a_{v}, a_{s}, e_{s y m}^{v}\right.$ and $\left.e_{s y m}^{s}\right)$ or $\left(f_{v}, f_{s}, f_{s y m}^{v}\right.$ and $\left.f_{s y m}^{s}\right)$. We leave here discussions on the temperature dependence of $a_{v}, f_{v}$ or $a_{s}, f_{s}$. They were discussed, for a different set of nuclear interactions in Ref. [38]. For the three Skyrme-class interactions used here, $a_{v}(T), f_{v}(T), a_{s}(T)$ and $f_{s}(T)$ follow nearly the same pattern.

In Fig. 7, the thermal evolution of the volume part of the symmetry energy coefficient $e_{s y m}^{v}$ (upper panels) and symmetry free energy coefficient $f_{s y m}^{v}$ (lower panels) for 


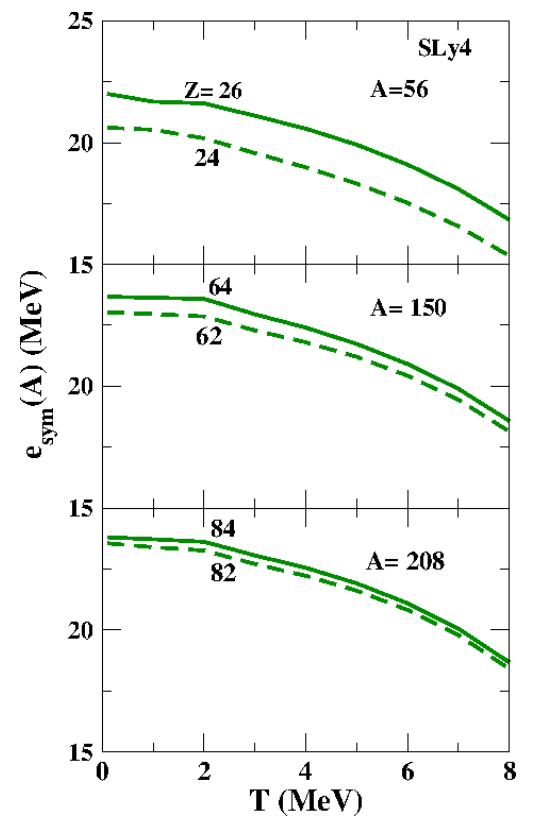

FIG. 4: (Color online) The temperature dependence of the symmetry coefficient $e_{\text {sym }}(A)$ calculated in the local density approximation (LDA, Eq. (34)) with the SLy4 interaction is shown for three nuclei with mass numbers $A=56,150$, and 208. The full lines correspond to calculations for isobars with the higher proton numbers, the dashed lines refer to those with proton numbers less by two.

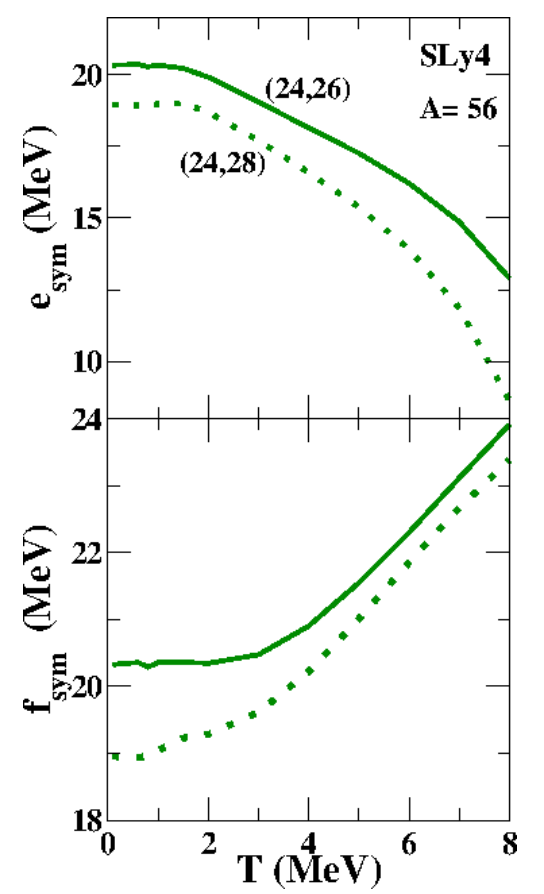

FIG. 5: (Color online) The temperature dependence of the symmetry coefficients $e_{\text {sym }}$ and $f_{\text {sym }}$ calculated in the difference method (Eq.(33)), shown for the nucleus $A=56$ with the SLy4 interaction. The full and dashed lines correspond to the isobars with different charge pairs. The charge numbers for the nuclear pair are given in the bracket.

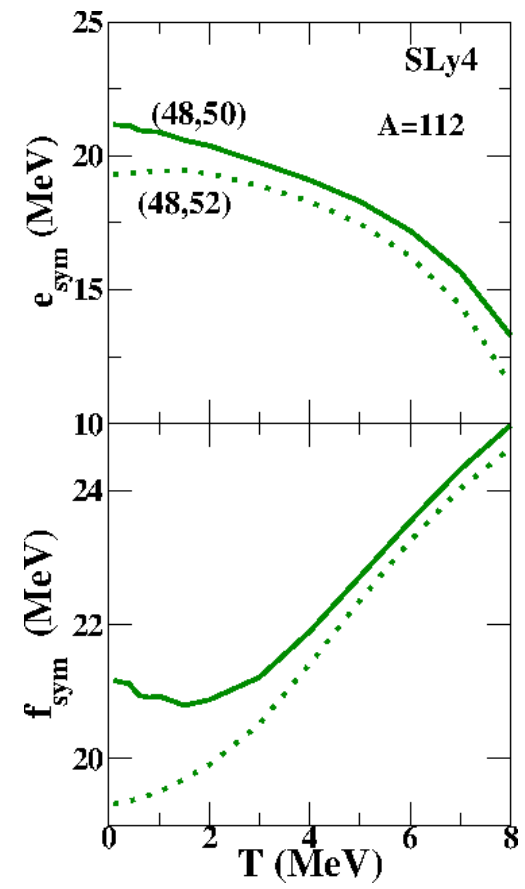

FIG. 6: (Color online) The same as in Fig. 5 for the nucleus with mass number $A=112$.

definition I, the right panels to definition II. The behavior of $e_{\text {sym }}^{v}$ depends on how $e_{\text {sym }}(A)$ is defined. In definition I, it generally falls with temperature (for SK255 interaction, the fall is slow though). In definition II, a slow increase in noticeable. The coefficient $f_{\text {sym }}^{v}$, on the contrary, shows a rise with temperature in definition I; in definition II, it is nearly temperature-independent.

The thermal dependence of the coefficients $\beta_{E}$ or $\beta_{F}$ for the three interactions is shown in the upper and lower panels of Fig. 8. At $T=0$, the value of $\beta_{E}$ or $\beta_{F}$ lies between $\sim 12-13 \mathrm{MeV}$ for the three interactions, in remarkable consonance with the empirical value of $\sim 13$ $\mathrm{MeV}$ obtained from the analyses of the 'experimental' symmetry energies of isobaric nuclei [54]. With temperature, $\beta_{E}$ decreases (for SK255 interaction, the decrease is again very slow), while $\beta_{F}$ generally increases. It is, however, noticed that both $e_{\text {sym }}^{v} / \beta_{E}$ and $f_{\text {sym }}^{v} / \beta_{F}$ are nearly temperature independent and also interaction independent in our calculations. They lie in the vicinity of $\sim$ $2.63 \pm 0.01$; this is very close to $\sim 2.64 \pm 0.01$ obtained earlier with the SBM and KDE0 interactions [38].

The temperature dependent surface symmetry coefficients $e_{\text {sym }}^{s}$ and $f_{\text {sym }}^{s}$ calculated using Eq. (38) in definition II are displayed in the upper and lower panels of Fig. 9. At $T=0, e_{\text {sym }}^{s}$ (also $f_{\text {sym }}^{s}$ ) is $\sim 41 \mathrm{MeV}$ for the $\mathrm{SkM}^{*}$ and SLy4 interactions, close to the value of $\sim$ $45 \mathrm{MeV}$ obtained by Stoitsov et al. [53], whereas for the SK255 interaction it is $\sim 58 \mathrm{MeV}$, nearly equal to that obtained from the binding energy analysis in Ref. [51]. As the temperature increases, $e_{s y m}^{s}$ increases sharply pointing to the growing importance of the surface in the cal- 


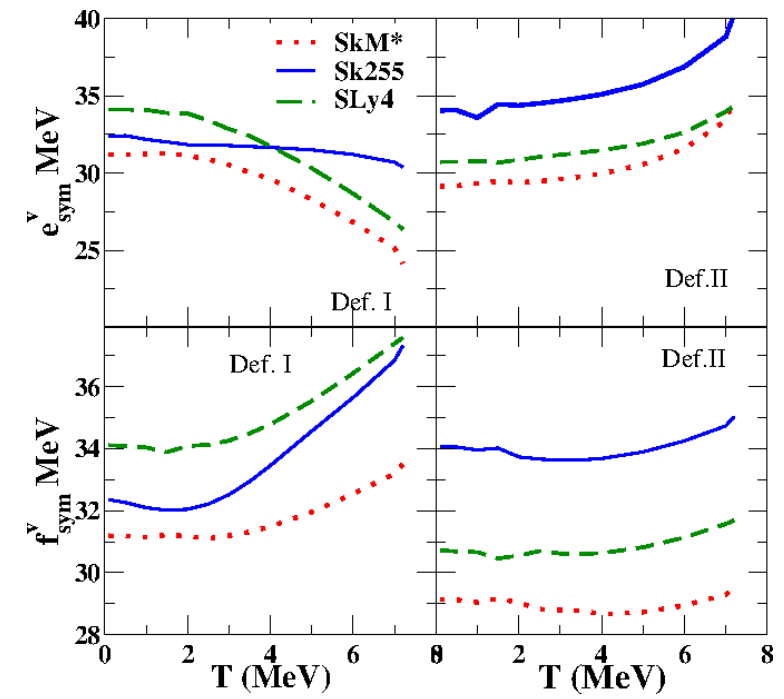

FIG. 7: (Color online) The volume symmetry energy coefficient $e_{\text {sym }}^{v}$ and symmetry free energy coefficient $f_{s y m}^{v}$, shown as a function of temperature for three interactions as displayed. The left panels present results calculated with the definition I (Eq. (37)), the right panels do so with the definition II (Eq. (38)).

slow decline with temperature for all the three interactions. Such a behavior was also noted in our earlier calculations with the SBM and KDE0 interactions 38]. A comparison of our results with those in Ref. 35 reveals that in both calculations, the surface symmetry coefficients are more temperature sensitive than the volume symmetry coefficients. However, in the calculations of [35], the temperature dependence of surface coefficients are comparatively more pronounced than those of ours. There are other subtle differences too; the density profiles used in 35] are not self-consistent. Also, high-density approximation in the whole temperature domain was used there.

Once the volume and surface symmetry coefficients are obtained as a function of temperature, the temperaturedependent symmetry coefficients for finite nuclei can be obtained unambiguously using definition I (Eqs. (37) and (41)) or definition II (Eqs. (38) and (42)) for $e_{\text {sym }}(A)$ and $f_{\text {sym }}(A)$. In Fig. 10, for two representative nuclei with $A=56$ and $A=208, e_{\text {sym }}(A)$ and $f_{\text {sym }}(A)$ are displayed as a function of temperature in the left and right panels, respectively, for the three interactions with the volume and surface symmetry coefficients so obtained. The full lines refer to $A=208$, the broken lines to $A=$ 56. The green color corresponds to definition I, the blue color to definition II. The general findings are: for all the three interactions, $e_{\text {sym }}(A)$ decreases with temperature, $f_{\text {sym }}(A)$ remains nearly unchanged upto $T \sim 3 \mathrm{MeV}$, and then increases slowly. To have a feel about the applicability of the local density approximation in calculating the thermal behaviour of the symmetry coefficients, re-

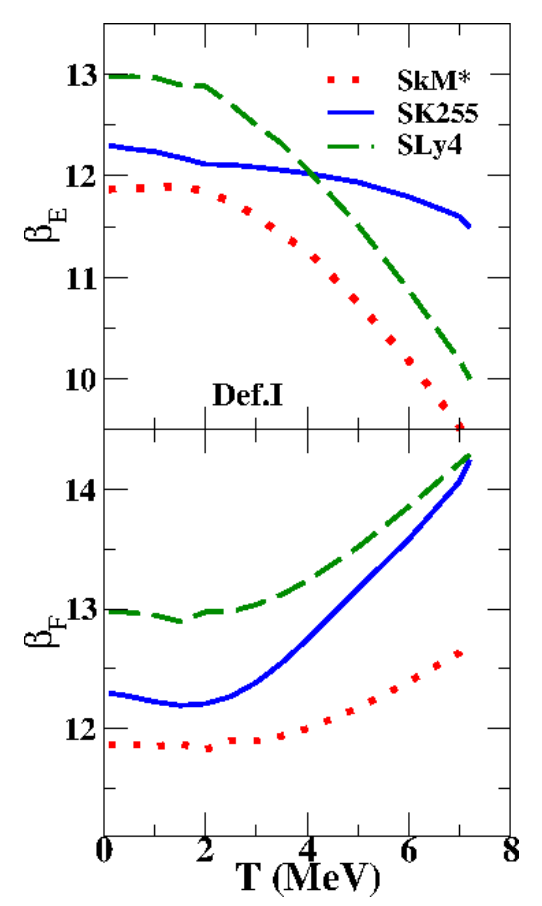

FIG. 8: (Color online) The thermal dependence of the coefficients $\beta_{E}$ and $\beta_{F}$ (from definition I, Eq. (37)) for the three interactions are shown.

corresponding to their mass numbers are also shown (in magenta color). In LDA, both $e_{\text {sym }}(A)$ and $f_{\text {sym }}(A)$ are seen to register a fall with increasing temperature.

The mass dependence of $e_{\text {sym }}(A)$ and $f_{\text {sym }}(A)$, calculated in definitions I and II is displayed in the left and right panels of Fig. 11 for the interactions at two temperatures, $T=0.0$ and $T=6.0 \mathrm{MeV}$. The green color again corresponds to definition I, the blue color refers to definition II. The dashed lines represent results at $T=0.0$, the full lines do so at $T=6.0 \mathrm{MeV}$. The general observations from Fig. 11 are that at fixed temperature, both $e_{\text {sym }}(A)$ and $f_{\text {sym }}(A)$ increase with $A$; this follows from the definition. It is also found that at a fixed mass number, $e_{\text {sym }}(A)$ decreases when temperature is raised whereas $f_{\text {sym }}(A)$ increases. These observations are true for both the definitions of the symmetry coefficients and collate with the already obtained understanding from Figs. 7, 8 and 9 on the thermal evolution of $f_{\text {sym }}^{v}, f_{\text {sym }}^{s}$, and $\beta_{F}$.

\section{CONCLUDING REMARKS}

Results from calculations on the temperature dependence of symmetry energy and symmetry free energy coefficients of infinite nuclear matter and also of finite nuclei, done in a finite-temperature-Thomas-Fermi model, are reported in this paper. Three Skyrme-class interactions are chosen, namely, SkM*, SLy4 and SK255. For infinite matter, we have made investigations at different densities, near saturation and also at densities in the sub- 


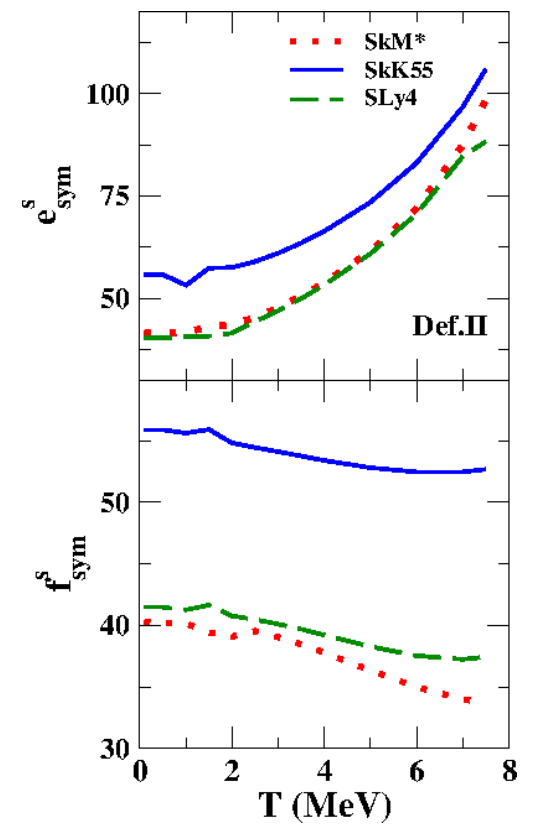

FIG. 9: (Color online) The thermal dependence of the surface symmetry coefficients $e_{\text {sym }}^{s}$ and $f_{\text {sym }}^{s}$ (from definition II, Eq. (38)) displayed for the three interactions.
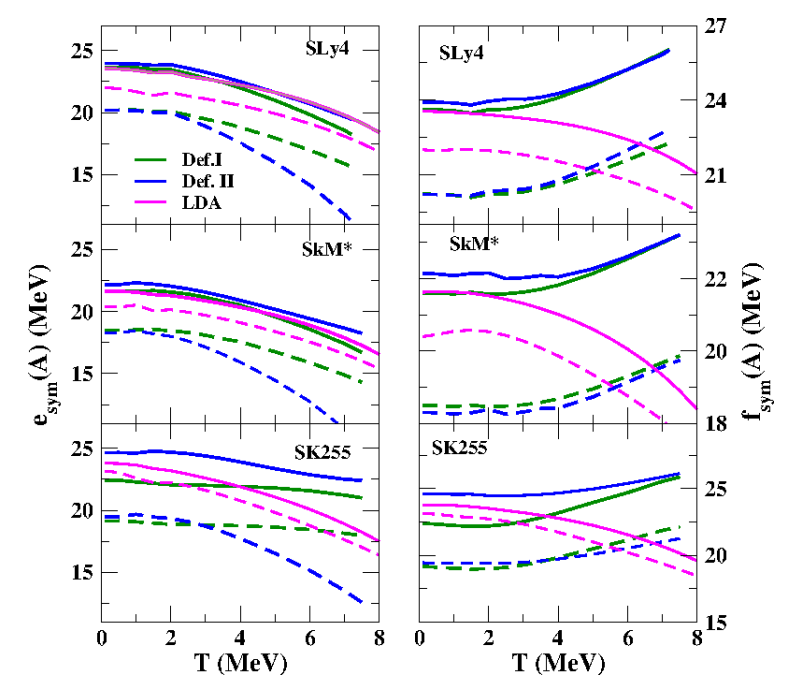

FIG. 10: (Color online) The temperature dependence of the symmetry coefficients of finite nuclei shown for the three interactions in definition I (Eq. (37), green line), definition II (Eq. (38), blue line) and in LDA (magenta line). The dashed lines correspond to calculations for $A=56$, the full lines refer to those for $A=208$. The left panels display results for $e_{\text {sym }}(A)$, the right panels do so for $f_{\text {sym }}(A)$. For more details, see text.

metry energy and symmetry free energy coefficients $e_{\text {sym }}$ and $f_{\text {sym }}$ show a very weak temperature dependence. At lower densities, however, $e_{\text {sym }}$ decreases with temperature whereas $f_{\text {sym }}$ displays a comparatively more prominent rise. For finite systems, in order to give stability to

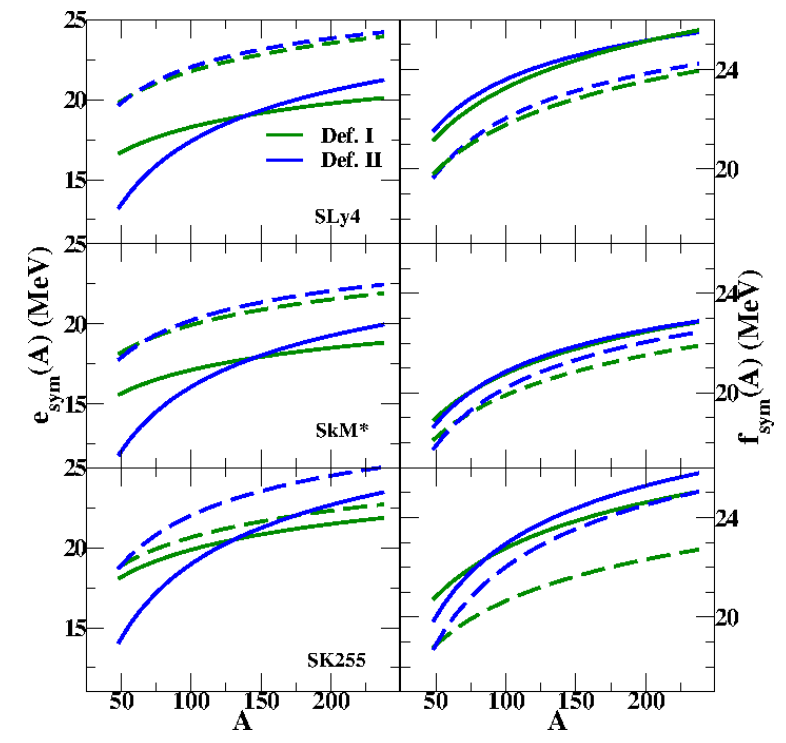

FIG. 11: (Color online) The mass number dependence of the symmetry coefficients $e_{\text {sym }}(A)$ (left panels) and $f_{\text {sym }}(A)$ (right panels) at two temperatures calculated with the three interactions. The green lines refer to def. I, the blue lines to def. II. The dashed lines correspond to results at $T=0.0$, the full lines do so at $T=6.0$.

TABLE I: Nuclear matter properties for the SkM*, SLy4 and SK255 interactions considered in this work.

\begin{tabular}{ccccccc}
\hline Force & $\begin{array}{c}e\left(\rho_{0}, 0,0\right) \\
(\mathrm{MeV})\end{array}$ & $\begin{array}{c}\rho_{0} \\
\left(\mathrm{fm}^{-3}\right)\end{array}$ & $\begin{array}{c}K_{v} \\
(\mathrm{MeV})\end{array}$ & $m^{*} / m$ & $\begin{array}{c}e_{\text {sym }} \\
(\mathrm{MeV})\end{array}$ & $\begin{array}{c}L \\
(\mathrm{MeV})\end{array}$ \\
\hline SK255 & 16.3 & 0.157 & 254.9 & 0.80 & 37.4 & 95 \\
SkM $^{*}$ & 15.8 & 0.160 & 216.6 & 0.79 & 30.0 & 46 \\
SLy4 & 16.0 & 0.160 & 229.9 & 0.70 & 32.0 & 46 \\
\hline
\end{tabular}

thermal Thomas-Fermi framework with subtraction. The Thomas-Fermi calculations are static in nature. Dynamical effects from the coupling of the surface phonons to the intrinsic particle motion are taken into account through a phenomenological parametric form of the nucleon energy mass $m_{\omega}$. At relatively low temperatures, the $\omega$-mass lends a heaviness to the nucleon effective mass; this has an appreciable effect on the symmetry coefficients. Once effects due to $\omega$-mass are taken into account, the symmetry coefficients are seen to decrease somewhat, particularly at low temperatures.

In understanding the symmetry coefficients $e_{\text {sym }}(A)$ and $f_{\text {sym }}(A)$ for finite nuclear systems and their thermal evolution, some ambiguities about their proper definition could be noted. We have explored the usual difference method [34], also the one obtained from the LDA [18]. In both these definitions, further specifications other than the mass number $A$ of the nucleus are necessary. In both these definitions, $e_{\text {sym }}(A)$ decreases with temperature. The symmetry free energy coefficient also falls with temperature in LDA, however, an opposite trend is observed 
TABLE II: The values of the Skyrme parameters for SkM*, SLy4 and SK255 interactions.

\begin{tabular}{lccc}
\hline \hline \multicolumn{1}{c}{ Parameters } & SkM $^{*}$ & SLy4 & SK255 \\
\hline$t_{0}\left(\mathrm{MeV} \mathrm{fm}^{3}\right)$ & -2645.0 & -2488.91 & -1689.35 \\
$t_{1}\left(\mathrm{MeV} \mathrm{fm}^{5}\right)$ & 410.0 & 486.82 & 389.30 \\
$t_{2}\left(\mathrm{MeV} \mathrm{fm}^{5}\right)$ & -135.0 & -546.39 & -126.07 \\
$t_{3}\left(\mathrm{MeV} \mathrm{fm}^{3(\alpha+1)}\right)$ & 15595.0 & 13777.0 & 10989.59 \\
$x_{0}$ & 0.09 & 0.834 & -0.1461 \\
$x_{1}$ & 0.0 & -0.344 & 0.116 \\
$x_{2}$ & 0.0 & -1.0 & 0.0012 \\
$x_{3}$ & 0.0 & 1.354 & -0.7449 \\
$\alpha$ & 0.1666 & 0.1666 & 0.3563 \\
\hline \hline
\end{tabular}

The ambiguities arising from these definitions of the symmetry coefficients of atomic nuclei are resolved from calculations of the symmetry energies and symmetry free energies of a host of nuclei along the periodic table as a function of temperature in the microscopic FTTF model with subtraction. The modification to the energies and free energies from the temperature-dependent energy mass are taken into account. The temperature dependence of the symmetry coefficients is then obtained from a liquid-drop-inspired fit of the total energies and free energies of these systems of nuclei. The two components, volume $\left(e_{\text {sym }}^{v}, f_{\text {sym }}^{v}\right)$ and surface $\left(e_{\text {sym }}^{s}, f_{\text {sym }}^{s}\right)$ that make up the total symmetry coefficients of finite systems have a temperature dependence that is nearly independent of the energy functionals chosen to calculate their energies. The volume symmetry energy coefficient $e_{\text {sym }}^{v}$ shows a strong temperature dependence, the surface part $e_{\text {sym }}^{s}$ displays an even stronger sensitivity. This results in a rapid fall of $e_{s y m}(A)$ as the temperature rises. The thermal sensitivity of the symmetry free energy coefficients is comparatively weaker. These calculations, in addition, give information on the thermal evolution of the volume and surface energies of nuclei. They are in excellent agreement with those in common usage, but because of the different focus in this communication are not reported here.

\section{Acknowledgments}

The authors gratefully acknowledge the assistance of Tanuja Agrawal in the preparation of the manuscript. J.N.D acknowledges the support of DST, Government of India. M.C. and X.V. acknowledge the support of the Consolider Ingenio 2010 Programme CPAN CSD200700042, of the grants FIS2011-24154 from MICINN and FEDER, and of grant 2009SGR-1289 from Generalitat de Catalunya.
[1] M. B. Tsang,T. X.Liu, L.Shi,P.Danielewicz,C. K. Gelbke,X. D.Liu,W. G.Lynch,W. P.Tan,G.Verde and A.Wagner Phys. Rev. Lett. 92, 062701 (2004).

[2] L.-W.Chen, C. M.Ko, and B.-A.Li, Phys. Rev. Lett. 94, 032701 (2005).

[3] B.-A.Li and L.-W.Chen Phys. Rev. C 72, 064611 (2005).

[4] B.-A.Li, L.-W.Chen and C. M.Ko Phys. Rep. 464, 113 (2008)

[5] M.Centelles, X.Roca-Maza, X. Viñas, and M. Warda, Phys. Rev. Lett. 102, 122502 (2009)

[6] U. Garg and et al Nucl. Phys. A788, 36 (2007)

[7] Li and et al Phys. Rev. Lett. 99, 162503 (2007)

[8] E. Baron, J. Cooperstein, and S. Kahana, Phys. Rev. Lett. 55, 126 (1985).

[9] A. W. Steiner, M. Prakash, J. M. Lattimer, and P. J. Ellis, Phys. Rep. 411, 325 (2005).

[10] H.-Th. Janka, K. Langanke, A. Marek, G. MartínezPinedo, and B. Müller, Phys. Rep. 442, 38 (2007).

[11] C. J. Horowitz and J. Piekarewicz, Phys. Rev. C 64, 062802 (2001).

[12] A. W. Steiner, Phys. Rev. C 77, 035805 (2008).

[13] L. F. Roberts, G. Shen, V. Cirigliano, J. A. Pons, S. Reddy, and S. E. Woosley, Phys. Rev. Lett. 108061103 (2012).

[14] M. Dutra, O. Lourenco J. S. Sà Martins, A. Delfinoi, J. R. Stone and P. D. Stevenson, Phys. Rev. C 85, 035201 (2012).

[15] B. K. Agrawal, J. N. De, and S. K. Samaddar, Phys. Rev. Lett. 109, 262501 (2012).
[16] J. Xu, L. W. Chen, B. A. Li, and H. R. Ma, Phys. Rev. C 75, 014607 (2007).

[17] J. N. De and S. K. Samaddar, Phys. Rev. C 85, 024310 (2012).

[18] S. K. Samaddar, J. N. De, X. Viñas, and M. Centelles, Phys. Rev. C 76, 041602 (R) (2007).

[19] Ch. C. Moustakidis, Phys. Rev. C 76, 025805 (2007).

[20] B. Friedman and V. R. Panpdharipande, Nucl. Phys. A 361, 502 (1981).

[21] G. Peilert, J. Randrup, H. Stocker, and W. Greiner, Phys. Lett. B 260, 271 (1991).

[22] C. J. Horowitz and A. Schwenk, Nucl. Phys. A 776, 55 (2006).

[23] E. OĊonnor, D. Gazit, C. J. Horowitz, A. Schwenk, and N. Barnea, Phys. Rev. C 75, 055803 (2007).

[24] S. Mallik, J. N. De, S. K. Samaddar, and S. Sarkar, Phys. Rev. C 77, 032201 (R), (2008).

[25] J. N. De and S. K. Samaddar, Phys. Rev. C 78, 065204 (2008).

[26] S. K. Samaddar, J. N. De, X. Viñas, and M. Centelles, Phys. Rev. C 80, 035803 (2009).

[27] J. N. De, S. K. Samaddar, and B. K. Agrawal, Phys. Rev. C 82, 045201 (2010).

[28] S. Kowalski et.al., Phys. Rev. C 75, 014601 (2007).

[29] J. B. Natowitz et. al., Phys. Rev. Lett. 104, 202501 (2010).

[30] J. N. De, S. K. Samaddar, X. Viñas, M. Centelles, I. N. Mishustin, and W. Greiner, Phys. Rev. C 86, 024601 (2012). 
[31] P. Donati, P. M. Poacher, P. F. Bortignon, and R. A. Broglia, Phys. Rev. Lett. 72, 2835 (1994).

[32] R. W. Hasse and P. Schuck, Nucl. Phys. A 445, 205 (1985).

[33] M. Prakash, J. Wambach, and Z. Y. Ma, Phys. Lett. B 128, 141 (1983).

[34] D. J. Dean, K. Langanke, and J. M. Sampaio, Phys. Rev. C 66, 045802 (2002).

[35] S. J. Lee and A. Z. Mekjian, Phys. Rev. C 82, 064319 (2010).

[36] J. N. De and S. K. Samaddar, Phys. Rev. C 85, 024310 (2012).

[37] E. Suraud, Nucl. Phys. A 462, 109 (1987).

[38] J. N. De, S. K. Samaddar, and B. K. Agrawal, Phys. Lett. B 716, 361 (2012).

[39] J. Bartel, P. Quentin, M. Brack, C. Guet, and H. B. Hakansson, Nucl. Phys. A 386, 79 (1982).

[40] E. Chabanat, P. Bonche, P. Haensel, J. Meyer, and R. Schaeffer, Nucl. Phys. A635, 231 (1998).

[41] B. K. Agrawal, S. Shlomo and V. Kim Au, Phys. Rev. C 68, 031304(R) (2003).

[42] P. Bonche, S. Levit, and D. vautherin, Nucl. Phys. A436, 265 (1985). S. Shlomo and J. B. Natowitz, Phys. Lett. B 252, 187 (1990).

[43] T. Sil, J. N. De, S. K. Samaddar, X. Viñas, M. Centelles, B. K. Agrawal, and S. K. Patra, Phys. Rev. C 66, 045803
(2002).

[44] J. N. De, S. Shlomo, and S. K. Samaddar, Phys. Rev. C 57, 1398 (1998).

[45] S. K. Samaddar, J. N. De, X. Viñas, and M. Centelles, Phys. Rev. C 75, 054608 (2007).

[46] S. Shlomo and J. B. Natowitz, Phys. Lett. B 252, 187 (1990).

[47] W. D. Myers and W. J. Swiatecki, Ann. Phys. (N. Y.) 55, 395 (1969).

[48] P.Danielewicz, Nucl. Phys. A727, 233 (2003).

[49] P. Danielewicz and J. Lee, Nucl. Phys. A 818, 36 (2009).

[50] E. Lipparini and S. Stringari, Phys. Lett. B112, 421 (1982).

[51] H. Jiang, G. J. Fu, Y. M. Zhao, and A. Arima, Phys. Rev. C 85, 024301 (2012).

[52] P.-G. Reinhard et. al, Phys. Rev. C 73014309 (2006).

[53] M. Stoitsov, R. B. Cakirli, R. F. Casten, W. Nazarewicz, and W. Satula, Phys. Rev. Lett. 98, 132502 (2007).

[54] M. Liu, N. Wang, Z. X. Li, and F. S. Zhang, Phys. Rev. C 82 (2010) 064306.

[55] J. N. De and S. K. Samaddar, Phys. Rev. C 78, 065204 (2008).

[56] P. Klüpfel, P.-G. Reinhard, T. J. Bürnevich, and J. A. Maruhn, Phys. Rev. C 79 (2009) 034310. 